\title{
Understanding the Role of Self-Judgment in the Association between Body Dissatisfaction and Quality of Life on Normal- Weight and Overweight Portuguese Women
}

\author{
Cláudia Ferreira, Patrícia Fortunato, Joana Marta-Simões and Inês A. Trindade \\ Universidade de Coimbra (Portugal)
}

\begin{abstract}
Literature has demonstrated the negative impact of body image dissatisfaction on women's quality of life. Nonetheless, it has been suggested that the relationship between body dissatisfaction and women's well-being is not linear, and that the processes that mediate this association remain unclear. This study aims to clarify the mediator role of self-judgment in the association between negative body image and psychological quality of life, in two groups: normal- weight and overweight women. This cross-sectional study comprised 200 normal-weight and 92 overweight female college students, aged between 18 and 24 years old, that completed self-report instruments of body dissatisfaction, self- judgment, and quality of life. Results showed that women who presented harsher self-judgment about their perceived failures tended to present lower levels in all quality of life domains. Also, results from mediation analyses indicated the relationship between body dissatisfaction and psychological quality of life was significantly mediated by the mecha- nisms of self-judgment in the two BMI groups (95\% CI [ -2.41 to -0.04$]$; $95 \%$ CI [ -6.35 to -.89$]$ ). This mediational model accounted for $28.3 \%$ and $40.7 \%$ of psychological quality of life in the normal-weight and overweight groups, respectively. These results suggest that a lower ability to deal with one's failures or inadequacies (e.g., negative evaluation of body image) in a kind and accepting manner may significantly increase the negative impact of body dissatisfaction on one's psychological quality of life. In this way, it seems that, the focus of interventions should go beyond body dissatisfaction and also target the development of adaptive attitudes (opposed to self-critical attitudes) to deal with negative body- related experiences.
\end{abstract}

Keywords: body dissatisfaction, quality of life, self-judgment, overweight, college women.

Recent research has pointed body image dissatisfaction as a pervasive phenomenon for women's well-being and mental health (Mond et al., 2013). Body image dis- satisfaction implies that one's weight and body shape are experienced as significantly different from the sociocultural feminine attractiveness standards (Stice \& Shaw, 2002). In Western societies these patterns of feminine attractiveness and beauty are overvalued by the media, and generally linked to control, success and happiness (Altabe \& Thompson, 1993; Sypeck et al., 2006). In accordance, excess of weight or obesity are nowadays highly associated with both negative percep- tions of body image and negative self-worth evaluations (e.g., Puhl \& Heuer, 2009). Nevertheless, body dissatisfaction is not exclusively experienced by overweight women; in contrast it has rather become the norm, given its prevalence even among women with normal body mass indexes (BMIs) (e.g., Rozin, Bauer, \&

Correspondence concerning this article should be addressed to Inês A. Trindade. CINEICC - Cognitive and Behavioral Centre for Research and Intervention. Faculty of Psychology and Education Sciences. University of Coimbra - (Portugal).

E-mail: ines.almeidatrindade@gmail.com
Catanese, 2003). In fact, it is undoubtedly known that body image dissatisfaction is a highly prevalent phe- nomenon for all BMI ranges. Mond and colleagues (2013) documented that almost $87 \%$ of women present some level of dissatisfaction concerning body size, shape and weight, and nearly $40 \%$ reported moderate to high levels of body dissatisfaction. These authors high- lighted that even though body dissatisfaction is a "normative" experience, it does not appear to be benign due to its link with concurrent and subsequent psychopathology (Mond et al., 2013).

In fact, body image dissatisfaction has been highly associated with a variety of negative health outcomes, such as depressive mood and eating psychopathology (e.g., Pinto-Gouveia, Ferreira, \& Duarte, 2014; Stice, Marti, \& Durant, 2011). Also, results from different studies emphasized the relationship between body dissatisfaction and quality of life $(\mathrm{QoL})$ andwell-being impairment, showing that higher body dissatisfaction was associated with decreased levels on all domains of QoL, especially psychosocial functioning (Duarte, Ferreira, Trindade, \& Pinto-Gouveia, 2015; Ferreira \& Trindade, 2015; Mond et al., 2013). In addition, the 
degree of mental health impairment has been shown to be proportional to the degree of body dissatisfaction (Mond et al., 2013). In accordance, research suggests that, when compared to those with normal weight $(18.5 \leq \mathrm{BMI}<25$ $\mathrm{kg} / \mathrm{m}^{2}$ ), individuals with excess weight or obesity (BMI $\geq$ $25 \mathrm{~kg} / \mathrm{m}^{2}$ ) present lower health- related QoL in all range of health dimensions (Kearns, Ara, Young, \& Relton, 2013).

Given the expressive prevalence of body dissatisfac- tion and its negative effect on various domains of QoL, evidence suggests that body dissatisfaction should be considered, by itself, as a relevant public health problem in Western societies, and a target for efforts in health promotion (Mond et al., 2013). Furthermore, research has generated support for the assertion that perceiving one's own body as divergent from the idealized body image does not directly impair the individual's QoL, and that this relationship may be mediated by relevant emotion regulation processes (Duarte et al., 2015). Specifically, even if some levels of body dissatisfaction are unavoidable for the majority of women, it is the way one replies to one's body dissatisfaction that can have a negative impact on one's health. Nonetheless, the adaptive and maladaptive emotion regulation strat- egies implicated in this relationship are less clear.

Self-compassion can be viewed as an adaptive emo- tion regulation strategy that holds a positive effect in mental health, in which painful or distressing feelings are not avoided but are instead held in awareness with kindness, understanding, and a sense of shared humanity (Neff, 2003a). According to Gilbert (2005), this emotion regulation process is characterized by a self-to-self relationship punctuated by a sense of care, warmth, and safeness. That is, feeling compassion for oneself involves accepting in a caring manner one's failures, imperfections or life's negative aspects (e.g., not being able to reach an ideal body shape or to avoid overeating; Gilbert, 2000). In fact, growing research shows that the ability to be selfcompassionate leads to the gentle acceptation of negative internal experiences (e.g., negative body image perceptions) with the feeling of connectedness, and to assume effective actions on promotion of one's well-being (Kelly, Vimalakanthan, \& Miller, 2014; Neff, 2004; Neff \& Vonk, 2009). Also, research has shown that self-compassion can buffer people against the impact of distressing and challenging situ- ations (Brion, Leary, \& Drabkin, 2014; Leary, Tate, Adams, Batts, \& Hancock, 2007). Namely, and in what concerns body image, a recent study showed that, for normal-weight college students, the harmful effect of negative experiences related to the body on QoL may be mitigated by the ability to be self-compassionate (Duarte et al., 2015). In contrast, recent studies have shown that body image dissatisfaction may become problematic when it becomes linked to maladaptive emotion regulation strategies (Pinto-Gouveia et al., 2014).

Literature has shown that women presenting selfjudgment about one's own failures or inadequacies, that over-identify themselves with one's own thoughts and emotion states, and that engage in feelings of isolation, present higher levels of distress and may become more vulnerable to psychopathology (Berry, Kowalski, Ferguson, \& McHugh,, 2010; Magnus, Kowalski, \& McHugh, 2010). Self-judgment is a mala- daptive strategy conceptualized as a critical self-to-self relationship which involves selfattribution of errors and unsuccessful life experiences, over identification with negative feelings when facing failure and being carried away by the story-line of one's own pain (Neff, 2003b). In fact, being harshly self-critical when facing setbacks or perceived inadequacies, or when perceiving some unwished characteristic about themselves, is often associated with decreases in psychological func- tioning, and with higher levels of negative mood and psychopathological symptoms, such as eating disor- dered symptomatology (Ferreira, Pinto-Gouveia, \& Duarte, 2013; Pinto-Gouveia et al., 2014). However, the role of this maladaptive emotion regulation in the relationship between body dissatisfaction and QoL remains unclear.

The current study aims are threefold. First, this study aims at understanding the differences between two different BMI categories (normal-weight and over- weight women) relatively to body dissatisfaction (BD), self-judgment, and subjective QoL regarding physical, psychological, relationship and environment domains. Second, this study intends to explore the associations between these variables both in normal-weight and overweight women. Given the links established between BMI, BD and impairment in emotional well-being, the insidious impact of $\mathrm{BD}$ on $\mathrm{QoL}$ might be expected to be most prominent for overweight women. Third, the main aim of this study is to examine whether self-judgment mediates the association between $\mathrm{BD}$ and women's subjective QoL, in normal and overweight women. We hypothesize that, for both normal weight and overweight women, the impact of a negative self- evaluation of body image on psychological QoL depends upon the extent to which individuals present selfjudgment.

\section{Methods}

\section{Procedure}

The present cross-sectional study is part of a wider research project about QoL and eating psychopathology in the Portuguese population. As in previous studies, the present paper's analyses were conducted in college women due to their well-known risk for presenting 
high levels of body image dissatisfaction and greater body image-related impairment (Luce, Crowther, \& Pole, 2008), specifically on psychological well-being.

First, the ethic committees of all educational institutions involved in the study provided their approval after the analysis of the present research protocols. Prior to the administration of the protocol, all participants gave their informed consent after being informed about the purpose, procedures, data confidentiality, and voluntary feature of their participation. Participants received extra credit for their participation. Then, the instruments were applied in the classroom context, taking into consideration the times scheduled with the enrolled educational institutions. The original sample consisted of 650 women. However, given the exclusion of participants due to non-correct completions of proto- cols (e.g., missing data superior to $15 \%$ in a question- naire), age requirements, and the study aims, the sample resulted in 292 participants, divided by two groups. The criterion used to distinguish the groups was participants' BMI: the overweight group comprised 92 women; while the normal-weight group was composed of 200 participants which were selected from a randomization of a wider group of normal-weight participants.

\section{Participants}

The sample of this study comprised two independent groups of female students, with ages ranging from 18 to 24 years old. The groups were defined by BMI. Therefore, 200 participants presented normal weight (BMI ranging from 18.5 to 24.9 ; WHO, 2000), with a mean age of 20.39 years old $(S D=1.64)$; and 92 partici- pants were overweight (BMI $\geq 25$; WHO, 2000), with a mean of 20.39 years of age $(S D=1.64)$. Regarding years of education, the two groups presented a mean of $13.28(S D=1.37)$ and 13.23 $(S D=1.35)$, respectively. The two groups did not present significant differences concern- ing age $t(290)=-.03 ; p=$ .976 , and years of education $t(290)=.30 ; p=.764$.

\section{Measures}

Body Mass Index (BMI)

BMI was calculated from participants' self-reported current height and weight using the Quetelet Index $\left(\mathrm{Kg} / \mathrm{m}^{2}\right)$.

Figure Rating Scale (FRS; Thompson \& Altabe, 1991; Portuguese validation by Ferreira, 2003)

The FRS is a well-known measure of body image dissatisfaction. It consists of a series of nine body silhou- ettes of different sizes, ranging from very thin (1) to very large (9). Participants respond by selecting the silhouettes that best represent their self-perceived current and ideal body images. The degree of body image dissatisfaction (BD) is estimated by the divergence between the two silhouettes. The scale has shown good temporal, convergent and divergent validities (Thompson \& Altabe, 1991).

Self-Compassion Scale (SCS; Neff, 2003a;

Portuguese validation by Castilho \& Pinto-Gouveia, 2011)

The SCS is a self-report instrument that assesses selfcompassion, designed to capture the respondent's selfperceived actions towards themselves during difficult times. The 26 items comprised in this instrument are rated on a Likert-type scale, ranging from " 1 " ("almost never") to "5" ("almost always"). The SCS comprises two main components: a positive one, including tree subscales: selfkindness, common humanity, and mindfulness subscales; and a negative one, comprising the self-judgment (e.g., "When I see aspects of myself that I don't like, I get down on myself"'), isolation (e.g., "When I fail at something that's important to me, I tend to feel alone in my failure"), and over-identification (e.g., "When something painful happens I tend to blow the incident out of proportion") subscales. For the pur- pose of this study, a composite measure of the 3 nega- tive dimensions of the SCS was computed and defined as a self-judgment dimension due to its association with the overstimulation of the threat system (Costa. Marôco, Pinto-Gouveia., Ferreira, \& Castilho, 2015). The SCS presented good internal reliability in the orig- inal version (.92) and in the Portuguese version (.89); in the present study, the Cronbach's alpha value was .79 for the normal-weight and .85 for overweight sample.

\section{World Health Organization Quality of Life - Bref (WHOQOL-BREF; WHOQOL Group, 1998; Portuguese validation by Canavarro, et al., 2006)}

The WHOQOL-BREF is a multidimensional and multicultural self-report measure that evaluates subjective QoL. It consists of twenty-six items, two of which are general and evaluate the global perception of health and QoL, and the other twenty-four items assess four broad domains: Physical Health (e.g., "To what extent do you feel that physical pain prevents you from doing what you need to do?") , Psychological Health (e.g., "To what extent do you feel your life to be meaningful?"), Social Relationships (e.g., "How satisfied are you with your personal relationships?"), and Environment (e.g., "How healthy is your physical environment?"). Items are scored on a fivepoint Likert-type scale, ranging from "1" ("extreme dissatisfaction") to "5" ("extreme satisfaction"), with higher scores indicating higher QoL. The WHOQOL-BREF showed adequate criterion and content validity, internal consistency and test-retest reliability, in both the original and the Portuguese 
versions. In this study, internal consistency values of .89 and .87 were obtained for the normal BMI group and the overweight group, respectively.

\section{Analytic Strategy}

The software SPSS (v.21 SPSS; Armonk, NY: IBM Corp) was used to perform data analyses. Independent sam- ples student's t-tests were used to explore the differ- ences between the variables of interest in both groups. Cohen's $d$ effect size was also computed. Pearson cor- relation coefficients were performed in both groups (Cohen, Cohen, West, \& Aiken, 2003), to analyse the relationships between body image dissatisfaction, self-judgement and QoL dimensions.

Finally, the mediation analyses were performed using PROCESS (Hayes, 2013), a macro that allows the establishment of path analyses. In the present study it were conducted two models of simple mediation (Model 4; Hayes, 2013) in the two BMI groups, exploring the indi- rect effect of body image dissatisfaction (independent variable) on psychological QoL (dependent variable) through the mechanism of self-judgment (mediator var- iable). The significance of the indirect effects between variables was tested using the bootstrap method (with 5000 samples), which produces a $95 \%$ confidence interval (bias-corrected and accelerated confidence intervals - $\mathrm{BCa}$ CIs) of the indirect effect. A statistically significant mediation effect exists if the values between the upper and lower confidence bound do not include zero.

\section{Results}

\section{Preliminary data analyses}

Uni and multivariate normality was examined by the values of Skewness (Sk) and Kurtosis (Ku), which indicated the absence of severe violation of normal distribution (Kline, 1998). In addition, preliminary data analyses indicated that these data were appropriate for regression analyses following the assumptions of normality, linearity, homoscedasticity, independence of errors, and multicollinearity (Field, 2004).

\section{Descriptives and differences between groups}

The means, standard deviations, and Student t-test results between the normal-weight sample $(n=200)$ and the overweight sample $(n=92)$ in all of the study variables, are reported in Table 1. Results showed that, consistently to what was expected, the two groups pre- sented significant differences with a large effect size regarding body dissatisfaction, with overweight par- ticipants presenting higher body dissatisfaction. Also, the groups presented differences in general, psychological and environmental QoL with medium effect sizes. Overweight participants revealed lower levels of these QoL dimensions when compared to normal-weight participants. However, these two groups did not differ concerning the physical and social relationships QoL domains, and in the maladaptive emotion regulation process in study (self-judgment).

\section{Correlations}

Pearson product-moment correlation coefficients (Table 2) indicated that BMI was positively associated with body image dissatisfaction (BD) for both samples. It is noteworthy to mention that BMI was signifi- cantly and negatively associated with general quality of life (QoL), but solely in the overweight sample. For both samples, BD was found to be correlated with self-judgment.

Table 1. Differences between the two groups (normal-weightand overweight women)

\begin{tabular}{|c|c|c|c|c|c|c|c|}
\hline & \multicolumn{2}{|c|}{$\begin{array}{l}\text { Normal-weight } \\
\text { sample }(n=200)\end{array}$} & \multicolumn{2}{|c|}{$\begin{array}{l}\text { Overweight sample } \\
(n=92)\end{array}$} & \multirow[b]{2}{*}{$t$} & \multirow[b]{2}{*}{$p$} & \multirow[b]{2}{*}{$d$} \\
\hline & $M$ & $S D$ & $M$ & $S D$ & & & \\
\hline BMI & 19.88 & 0.73 & 27.15 & 2.08 & -32.64 & $* * *$ & 4.66 \\
\hline $\mathrm{BD}$ & 0.25 & 0.87 & 1.55 & 0.72 & -12.52 & $* * *$ & 1.63 \\
\hline Self-judgment & 2.69 & 0.78 & 2.69 & 0.89 & 0.02 & .985 & \\
\hline Physical QoL & 75.55 & 11.27 & 74.07 & 10.87 & 1.06 & .291 & \\
\hline Psychological QoL & 70.50 & 13.27 & 64.95 & 14.11 & 3.26 & $* *$ & 0.41 \\
\hline Relationships QoL & 75.21 & 16.68 & 72.01 & 15.70 & 1.55 & .122 & \\
\hline Environmental QoL & 69.50 & 11.59 & 65.35 & 10.71 & 2.91 & $* *$ & 0.37 \\
\hline General QoL & 75.37 & 12.14 & 67.66 & 13.51 & 4.86 & $* * *$ & 0.60 \\
\hline
\end{tabular}

Note

$* * p<.010 ; * * * p<.001 ;$

BMI = Body Mass Index; BD = Body Image Dissatisfaction; Self-jugment $=$ dimension of SCS; Physical QoL, Psychological QoL, Relationships QoL, Environmental QoL, General QoL = domains of the WHOQOL_BREF. 
Table 2. Correlations between study variables in the normal-weight $(N W ; n=200)$ and overweight $(\boldsymbol{O W} ; \boldsymbol{n}=\mathbf{9 2} ; \boldsymbol{i n}$ bold $)$ groups

\begin{tabular}{|c|c|c|c|c|c|c|c|c|}
\hline & 1 & 2 & 3 & 4 & 5 & 6 & 7 & 8 \\
\hline 1.BMI & - & $.25^{*}$ & -.01 & .04 & .02 & .15 & -.15 & $-.24 *$ \\
\hline 2.BD & $.23 * *$ & - & $.29 *$ & $-.22 *$ & $-.33 * *$ & $-.27 * *$ & -.15 & $-.29 * *$ \\
\hline 3.Self-Judgment & -.09 & $.16^{*}$ & - & $-.36 * * *$ & $-.62 * * *$ & $-.33 * *$ & -.14 & $-.32 * *$ \\
\hline 4. Physical QoL & -.07 & $-.16^{*}$ & $-.37 * * *$ & - & $.63 * * *$ & $.39 * * *$ & $.46 * * *$ & $.56 * * *$ \\
\hline 5. Psychological QoL & -.06 & $-.19 * *$ & $-.52 * * *$ & $.60 * * *$ & - & $.57 * * *$ & $.38 * * *$ & $.51 * * *$ \\
\hline 6. Relationships QoL & -.09 & -.09 & $-.38 * * *$ & $.38 * * *$ & $.56 * * *$ & - & $.28 * *$ & $.18^{*}$ \\
\hline 7. Environmental QoL & -.05 & -.03 & $-.23 * * *$ & $.53 * * *$ & $.55 * * *$ & $.34 * *$ & - & $.50 * * *$ \\
\hline 8. General QoL & -.06 & -.12 & $-.21 * *$ & $.39 * * *$ & $.42 * * *$ & $.29 * *$ & $.50 * * *$ & - \\
\hline
\end{tabular}

Note

$* p<.050 ; * * p<.010 ; * * * p<.001 ;$

BMI = Body Mass Index; BD = Body Image Dissatisfaction; Self-jugment $=$ dimension of SCS; Physical QoL, Psychological QoL, Relationships QoL, Environmental QoL, General QoL = domains of the WHOQOL_BREF.

Additionally, in the overweight sample, BD showed negative and significant associations with general QoL and with the physical, psychological and social rela- tionships QoL dimensions; although an exception was verified regarding the environmental dimension of QoL. In the normal-weight sample, BD only presented significant, although weak, negative correlations with physical and psychological QoL dimensions.

In both samples it were evidenced negative associa- tions between self-judgment and all QoL domains and overall QoL. An exception was verified in the over- weight sample regarding the environmental dimension of QoL, which revealed a non-significant association with this maladaptive emotional process.

\section{Mediation analyses}

With the purpose of exploring the mediator role of selfjudgment in the association between body image dissatisfaction (BD) and psychological QoL, two medi- ator analyses (Hayes, 2013) were conducted in the normalweight female sample (Figure 1) and in the overweight female sample (Figure 2).
In the normal-weight sample, the individual analysis of the relationships between variables revealed that $\mathrm{BD}$ is significantly associated with self-judgment $(b=0.14 ; S E=$ $0.06 ; p=.028)$ in a significant model $(F(1,198)=4.92 ; p=$ .028). Furthermore, psychological QoL was associated with self-judgment $(b=-8.51 ; S E=1.04 ; \quad p<.001)$ but not with body dissatisfaction $(b=-1.75 ; S E=0.93 ; p=.060)$ in a model that explained $28.3 \%$ of psychological QoL $(F(2$, $197)=38.87 ; p<.001$ ).

Despite this non-significant direct effect, it was found an indirect effect in the relationship between body dissatisfaction and psychological QoL through selfjudgment $b=-1.19 ; S E=0.59 ; 95 \%$ CI [ -2.41 to -0.04$]$.

Therefore, the negative effect of body dissatisfaction on the psychological QoL of normal-weight women seemed to act through the mechanisms of self-judgment. Regarding the overweight sample, the same proce- dure was conducted. The individual analysis of the associations between variables demonstrated that $\mathrm{BD}$ is significantly associated with self-judgment $(b=0.36 ; S E=0.13 ; p=.006)$ in a significant model $(F(1,90)=8.00 ; p=.006)$. Moreover, in this group, psychological QoL was also associated with self-judgment $(b=-9.05$;

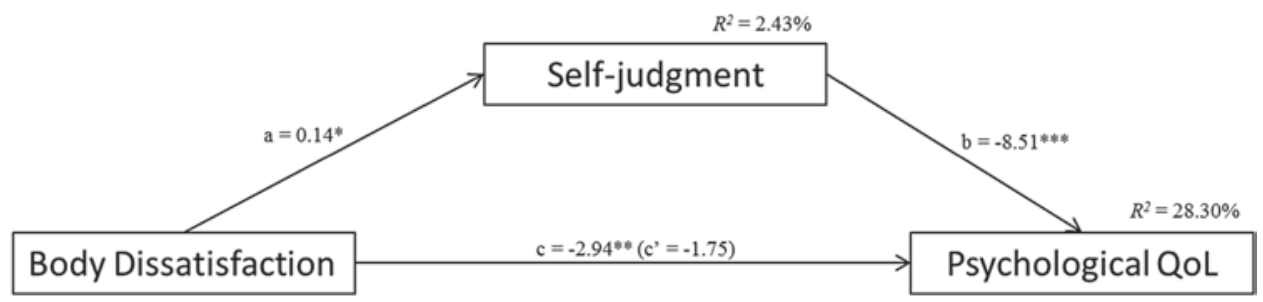

Figure 1. Normal-weight sample.

Note: Visual representation of the simple mediation in the normal-weight group $(n=200)$ exploring the effect of self-judgment on the association between body dissatisfaction and psychological QoL. The values on the arrows represent non-standardized regression coefficients. $\mathrm{a}=$ the effect of body dissatisfaction on self-judgment; $\mathrm{b}=$ the effect of self-judgment on psychological QoL; $\mathrm{c}=$ the total effect of body dissatisfaction on psychological QoL; c' = the direct effect of body dissatisfaction on psychological QoL after the inclusion of the mediator variable (self-judgment).

$* p<.05, * * p<.01, * * * p<.001$. 


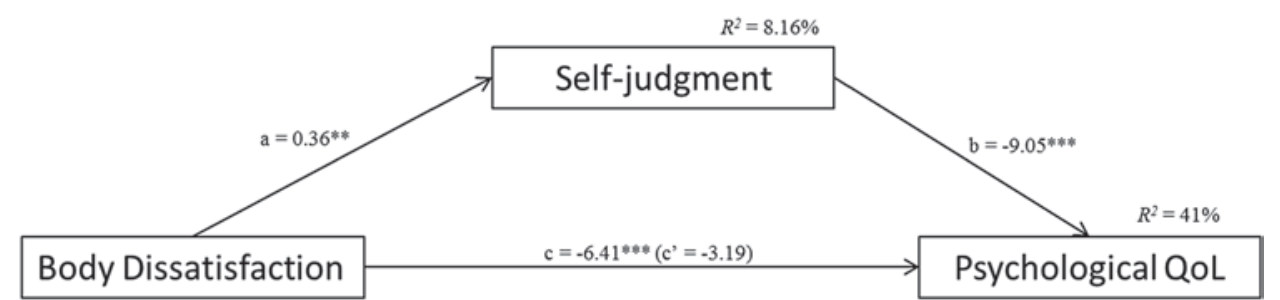

Figure 2. Overweight sample.

Note: Visual representation of the simple mediation in the overweight group $(n=98)$ exploring the effect of self-judgment on the association between body dissatisfaction and psychological QoL. The values on the arrows represent non-standardized regression coefficients. $\mathrm{a}=$ the effect of body dissatisfaction on self-judgment; $\mathrm{b}=$ the effect of self-judgment on psychological QoL; $\mathrm{c}=$ the total effect of body dissatisfaction on psychological QoL; c' = the direct effect of body dissatisfaction on psychological QoL after the inclusion of the mediator variable (self-judgment).

$* p<.05, * * p<.01, * * * p<.001$.

$S E=1.35 ; p<.001)$ but not with body dissatisfaction $(b=$ $-3.19 ; S E=1.68 ; p=.060)$ in a model that explains $41 \%$ of psychological QoL $(F(2,89)=30.53 ; p<.001)$.

It was also found an indirect effect in the association between body dissatisfaction and psychological QoL through self-judgment $(b=-3.22 ; S E=1.39 ; 95 \%$ CI $[-$ 6.35 to -.89$]$. These results seem to indicate that the negative effect of body dissatisfaction on overweight women's psychological QoL also seems to be mediated by self-judgment.

\section{Discussion}

Previous research suggested that the negative impact of body image dissatisfaction on women's mental well-being is not linear (Mond et al., 2013). Specifically, although it is reported that some levels of body dissat- isfaction are unavoidable for the majority of women, it may be the way one replies to body dissatisfaction that can have a negative impact on one's psychological health. Nonetheless, the maladaptive emotion regula- tion strategies implicated in this relationship remain unclear.

The main aim of this study was therefore to test the mediator effect of self-judgment on the relationship between the negative experience of one's own body image and psychological QoL, in normal-weight and overweight female students. For this purpose, statis- tical analyses were performed in two independent samples: normal-weight group $(18.5 \leq \mathrm{BMI} \leq 24.9)$ and overweight group (BMI $\geq$ 25).

Comparisons analyses from the BMI groups showed, as expected, that overweight women presented higher levels of body image dissatisfaction, when compared to normalweight participants. Additionally, and in accordance with previous research (e.g., Kearns et al., 2013), female students with excess of weight or obesity, when compared to those with normal-weight presented lower levels of an overall perception of well-being.
Also, overweight women reported lower levels in psychological and environmental QoL domains. However, these two groups did not present significant differences concerning physical and social relationships QoL domains, and also self-judgment.

In line with extant literature, this study showed that BMI was positively linked to body image dissatisfac- tion, in the normal-weight and the overweight groups (e.g., Puhl \& Heuer, 2009; Rozin, Bauer, \& Catanese, 2003). Interestedly, BMI did not present significant associations with the dimensions of QoL, neither for normal and overweight female college students. An exception was verified in the overweight group, in which BMI was inversely correlated with general QoL. Regarding the relationship between body image dis- satisfaction and QoL domains, the present results go in line with previous research (Kearns et al., 2013). Specifically, results demonstrated that for overweight and obese female students a negative perception of body image was linked to low levels of overall well-being and to physical, psychological and social QoL. While in the normal-weight group body image dissatisfac- tion only presented significant, although weak, nega- tive correlations with physical and psychological QoL dimensions.

Concerning the relationship between self-judgment and QoL, the present study extended previous research which has demonstrated the link between this emotion regulation process and psychopathology (Berry et al., 2010; Ferreira et al., 2013; Magnus et al., 2010; Pinto- Gouveia et al., 2014). Specifically, results showed that women who presented harsher self-judgment about their failures or inadequacies, over-identifying them- selves with thoughts and emotional states, and pre- senting feelings of isolation, tend to present lower levels in overall and all QoL domains. In fact, a harsh self-critical attitude was associated with poorer physical, psychological and social QoL indices in both groups. An exception was verified regarding the environmental 
dimension of QoL, which revealed a non-significant association with this maladaptive emotional process in the overweight sample. It seems important to note that, in the overweight sample, self-judgment showed a strong negative association with psychological QoL, which is considered a main aspect to define individ- uals' overall level of health (Camfield \& Skevington, 2008; Hoffman \& Driscoll, 2000).

Additionally, to further understand the relationship between body image dissatisfaction, psychological QoL and self-judgment in normal-weight and overweight female college students, mediation analyses were con- ducted for each group. Results indicated that, in both BMI groups, body image dissatisfaction presented a negative effect on psychological QoL with a simulta- neous indirect effect through the mechanism of self- judgment. This mediational model explained a total of $28.3 \%$ and $40.7 \%$ of the variance of psychological QoLin the normal-weight and overweight group, respectively. These findings suggest that the experience of body dissatisfaction by normal-weight and overweight women is linked to a higher engagement in an emotion regulation process marked by criticism and judgment towards the self, which seems to explain lower psycho- logical QoL. These findings may indicate that the extent to which negative body-related experiences impact on women's well-being depends on the level of selfjudgment, i.e., a critical self-to-self relationship which involves self-attribution of error and unsuc- cessful life events. It was interesting to verify that the same impact pattern was observed in normal-weight women and women with excess of weight. These data seem to show that the way one responds to body-related experiences significantly influence the impact of those experiences on psychological QoL, but. In other words, data suggests that the experience of physical appear- ance dissatisfaction, when mediated by judgmental and self-critical attitudes, results in worse psychological

QoL indicators.

These findings cannot be considered without taking account some limitations. Firstly, the main limitation of the present study is its cross-sectional nature which does not allow causal interpretations. Therefore, future research should focus on longitudinal and experimental research of emotion regulation processes' role on women's QoL. Secondly, the tested mediational model is restrained since other emotion regulation processes are probably involved in the association between body image dissatisfaction and QoL (e.g., body image flexi- bility, rumination). In this study, the mediational model was intentionally limited to specifically explore the impact of self-judgment on QoL. Nevertheless, in future studies, different emotion processes should be tested. Finally, another possible limitation concerns the char- acteristics of the sample. The use of a convenience sample of female college students can limit the generalization of the data. Indeed, future studies should test our findings in more diversified samples, with different age ranges, different cultures, and gender.

Nevertheless, the present study seems to contribute to the growing literature concerning the role of self- judgment in the relationship between body image dis- satisfaction and psychological QoL in normal-weight and overweight women. It was indeed interesting to note that body dissatisfaction might not directly impact women's psychological health, which rather seems to depend upon the extent to which individuals present negative selfjudgment and self-scrutiny when facing inadequacies or unwished characteristics about them- selves. These findings may contribute to future research and may have important implications for the develop- ment of community-based interventions focused on the promotion of QoL in women. In particular, this study seems to emphasize that these interventions should target not only one's experience related to body image, but mostly how one relates to such experience. That is, our findings suggest that rather than efforts being placed in targeting weight or body image dissat- isfaction, interventions should focus on developing adaptive emotion regulation, opposed to harsh self- critical and judgmental attitudes, to deal with such negative experiences related to body image.

\section{References}

Altabe M., \& Thompson J. K. (1993). Body image changes during early adulthood. International Journal of Eating Disorders, 13, 323-328.

Berry K., Kowalski K. C., Ferguson L. J., \& McHugh T. F. (2010). An empirical phenomenology of young adult women exercisers' body self-compassion. Qualitative Research in Sport and Exercise, 2, 293-312.

Brion J. M., Leary M. R., \& Drabkin A. S. (2014).

Self-compassion and reactions to serious illness: The case of HIV. Journal of Health Psychology, 19, 218-229. http://dx.doi.org/10.1177/1359105312467391

Camfield L., \& Skevington S. M. (2008). On subjective wellbeing and quality of life. Journal of Health Psychology, 13, 764-775. http://dx .doi.org/10.1177/1359105308093860

Canavarro M. C., Simões M. R., Serra A. V., Pereira M., Rijo D., Quartilho M.,... Carona C. (2006). WHOQOL-Bref Instrumento de Avaliação da Qualidade de Vida da Organização Mundial de Saúde [WHOQOL-Bref Quality of Life Assessment Instrument from the World Health Organization]. In M. R. Simões, M. M. Gonçalves \& L. S. Almeida (Coord.), Avaliação Psicológica: 46 Instrumentos válidos para a população portuguesa [Psychological Assessment: 46 valid instruments for the Portuguese population] (Vol. III, pp. 77-100). Coimbra, Portugal: Quarteto.

Castilho P., \& Pinto-Gouveia J. (2011). Auto-Compaixão: Estudo da validação da Versão Portuguesa da Escala de AutoCompaixão e da sua relação com as experiências adversas na infância, a comparação social e a 
psicopatologia [Self-compassion: Validation study of the Portuguese version of the Self-Compassion Scale and its association with early adverse experiences, social comparison and psychopathology]. Psychologica,

54, 203-229.

Cohen J., Cohen P., West S., \& Aiken L. (2003). Applied multiple regression/correlation analysis for the behavioral sciences ( $3^{\text {th }}$ Ed.), New Jersey, NJ: Lawrence Erlbaum Associates.

Costa J.,MarôcoJ.,Pinto-Gouveia J.,Ferreira C., \& Castilho P (2015). Validation of the psychometric properties of the SelfCompassion Scale. Testing the factorial validity and factorial invariance of the measure among borderline personality disorder, anxiety disorder, eating disorderand general populations. Clinical Psychology and Psychotherapy. http://dx.doi.org/10.1002/cpp.1974

Duarte C., Ferreira C., Trindade I. A., \& Pinto-Gouveia J (2015). Body image and college women's quality of life: The importance of being self-compassionate. Journal of Health Psychology, 20, 754-764.

Ferreira C. (2003). Anorexia Nervosa: A expressão visível do invisível. Contributos para a avaliação de atitudes $e$ comportamentos em relação ao peso e à imagem corporal

[Anorexia nervosa: The visible expression of the invisible. Contributions for the assessment of attitudes and behaviors in relation to weight and body image]. Unpublished master's thesis. University of Coimbra. Coimbra, Portugal.

Ferreira C., \& Trindade I.A. (2015). Body image-related cognitive fusion as a main mediational process between body-related experiences and women's quality of life. Eating and Weight Disorders, 20(1),91-97.http://dx.doi. org/10.1007/s40519-014-0155-y

Ferreira C., Pinto-Gouveia J., \& Duarte C. (2013). Self-compassion in the face of shame and body image dissatisfaction: implications for eating disorders. Eating Behaviors, 14, 207-210. http://dx.doi.org/10.1016/ j.eatbeh.2013.01.005

Field A. (2004). Discovering statistics using SPSS ( $\left.3^{\text {th }} \mathrm{Ed}\right)$. London, UK: Sage Publications.

Gilbert P. (2000). The relationship of shame, social anxiety and depression: The role of the evaluation of social rank. Clinical Psychology \& Psychotherapy, 7, 174-189. http://dx. doi.org/10.1002/1099-0879(200007)7:3<174::AID-CPP236> 3.0.CO;2-U

Gilbert P. (2005). Social mentalities: A biopsychosocial and evolutionary reflection on social relationships.

In M. W.Baldwin (Ed.), Interpersonal cognition (pp. 299-335). New York, NY: Guilford.

Hayes A. F. (2013). Introduction to mediation, moderation, and conditional process analysis: A regression-based approach. New York, NY: The Guilford Press.

Hoffman M., \& Driscoll J. (2000). Health promotion and disease prevention: A concentric biopsychosocial model of health status. In S. D. Brown \& R. W.Lent RW (Eds.), Handbook of counseling psychology. (pp. 532-570). New York, NY: John Wiley

Kearns B., Ara R., Young T., \& Relton C. (2013). Association between body mass index and health-related quality of life, and the impact of self-reported long-term conditions - cross-sectional study from the south Yorkshire cohort dataset. BMC Public Health, 13, 1009. http://dx.doi.org/10.1186/1471-2458-13-1009

Kelly A. C., Vimalakanthan K., \& Miller K. E. (2014). Self-compassion moderates the relationship between body mass index and both eating disorder pathology and body image flexibility. Body Image, 11, 446-453. http://dx.doi. org/10.1016/j.bodyim.2014.07.005

Kline R. (1998). Principles and practice of structural equation modeling. New York, NY: The Guilford Press.

Leary M. R., Tate E. B., Adams C. E., Batts A. A., \& Hancock J (2007). Self compassion and reactions to unpleasant self-relevant events: The implications of treating oneself kindly. Journal of Personality and Social Psychology, 92 887-904. http://dx.doi.org/10.1037/0022-3514.92.5.887

Luce K. H., Crowther J. H., \& Pole M. (2008). Eating Disorder Examination Questionnaire (EDE-Q): Norms for undergraduate women. International Journal of Eating Disorders, 41, 273-276. http://dx.doi.org/10.1002/eat.20504

Magnus C. M. R., Kowalski K. C., \& McHugh T. L. F. (2010). The role of self-compassion in women's self- determined motives to exercise and exercise-related outcomes. Self and Identity, 9, 363-382. http://dx.doi.org/ 10.1080/15298860903135073

Mond J., Mitchison D., Latner J., Hay P., Owen C., \& Rodgers B. (2013). Quality of life impairment associated with body dissatisfaction in a general population sample of women. BMC Public Health, 13, 920. http://dx.doi.org/ 10.1186/14712458-13-920

Neff K.D. (2003a). Development and validation of a scale to measure self-compassion. Self and Identity, 2, 223-250. http://dx.doi.org/10.1080/15298860309027

Neff K. D. (2003b). Self-compassion: An alternative conceptualization of a healthy attitude toward oneself. Self and Identity , 2, 85-101. http://dx.doi.org/10.1080/ 15298860309032

Neff K. D. (2004). Self-compassion and psychological well-being. Constructivism in the Human Sciences, 9, 27-37.

Neff K. D., \& Vonk R. (2009). Self-compassion versus global self-esteem: Two different ways of relating to oneself. Journal of Personality, 77, 23-50. http://dx.doi.org/ 10.1111/j.1467-6494.2008.00537.x

Pinto-Gouveia J.,Ferreira C.,\& Duarte C. (2014). Thinnes in the pursuit for social safeness: An integrative model of social rank mentality to explain eating psychopathology. Clinical Psychology \& Psychotherapy, 21, 154-165. http://dx.doi.org/10.1002/cpp.1820

Puhl R. M., \& Heuer C. A. (2009). The stigma of obesity: A review and update. Obesity, 17,941-964.http://dx.doi org/10.1038/oby.2008.636

Rozin P., Bauer R., \& Catanese D. (2003). Attitudes to food and eating in American college students in six different regions of the United States. Journal of Personality \& Social Psychology, 85(1), 132-141. http://dx.doi.org/10.1037/

0022-3514.85.1.132

Stice E., \& Shaw H. E. (2002). Role of body dissatisfaction in the onset and maintenance of eating pathology: A synthesis of research findings. Journal of Psychosomatic Research, 53,985-993. http://dx.doi.org/10.1016/ S00223999(02)00488-9 
Stice E., Marti C. N., \& Durant S. (2011). Risk factors for onset of eating disorders: Evidence of multiple risk pathways from an 8-year prospective study. Behaviour Research and Therapy, 49, 622-627. http://dx.doi.org/

10.1016/j.brat.2011.06.009

Sypeck M. F., Gray J. J., Etu S. F., Ahrens A. H.,

Mosimann J. E., \& Wiseman C. V. (2006). Cultural

representations of thinness in women, redux: Playboy

magazine's depictions of beauty from 1979 to 1999 . Body

Image: An International Journal of Research, 3, 229-235.

http://dx.doi.org/10.1016/j.bodyim.2006.07.001
Thompson J. K., \& Altabe M. N. (1991). Psychometric qualities of the figure rating scale. International Journal of Eating Disorders, 10, 615-619. http://dx.doi.org/ 10.1002/1098-108X(199109)10:5\%3C615::

AID-EAT2260100514\%3E3.0.CO;2-K

WHOQOL GROUP (1998). Development of the World Health Organization WHOQOL-BREF Quality of Life Assessment. Psychological Medicine, 28, 551-558.

WHO (2000). The World Health Report 2000. Obesity - Preventing and managing the global epidemic. Geneva. Switzerland: Author. 